\title{
Mapeamento Ontológico para Classificar Mensagens Significativas em um Ambiente Colaborativo de Aprendizagem em Estatística
}

\author{
Andressa Oliveira Souza - UTFPR - andressasouza@alunos.utfpr.edu.br \\ Simone Nasser Matos - UTFPR - snasser@utfpr.edu.br \\ Eliana Cláudia Mayumi Ishikawa - UTFPR - eishikawa@utfpr.edu.br \\ Helyane Bronoski Borges - UTFPR - helyane@utfpr.edu.br
}

\begin{abstract}
RESUMO: Este artigo descreve um mapeamento ontológico para avaliar mensagens significativas entre indivíduos em um ambiente colaborativo de atividades. A ontologia proposta foi aplicada nas mensagens trocadas entre os participantes do Collabora, que é um ambiente de aprendizagem colaborativa para promover a colaboração entre os alunos por meio de um chat e permite a troca de mensagens, links e arquivos para a resolução de atividades. Como resultado o mapeamento proposto permitiu a identificação das mensagens relacionadas ao domínio, a afetividade e valorização de autonomia.
\end{abstract}

Palavras-chaves: Colaboração. Avaliação de Mensagens. Ontologias.

ABSTRACT: This paper describes an ontological mapping to evaluate meaningful messages between individuals in a collaborative activity environment. The proposal ontology was applied on messages sent between the students of Collabora, that is a collaborative learning environment to promote the collaboration between the students through of a chat and allows an exchange of messages, links and files for a resolution activity. As a result, the proposed mapping allowed the identification of messages related to affectivity, autonomy valorization and domain.

Keywords: Colaboration. Message Evaluation. Ontology.

\section{Introdução}

A Aprendizagem Colaborativa Apoiada por Computadores (Computer Supported Collaborative Learning - CSCL) estuda como as pessoas podem aprender em grupo com o auxílio do computador, podendo ser aplicada em todos os níveis da educação (STAHL et al., 2006).

A CSCL dispõe de recursos computacionais como ferramentas mediadoras do processo de ensino-aprendizagem do grupo. O suporte dado pelo computador à aprendizagem colaborativa, por meio dessas ferramentas, visa melhorar a dinâmica entre os membros do grupo, possibilitando aos mesmos alcançarem objetivos mais ricos em conteúdo, na medida em que reúne propostas e soluções de vários alunos do grupo, o que incentiva o compartilhamento de informações e a responsabilidade sobre sua veracidade.

Com os avanços das tecnologias de comunicação das últimas décadas, tal como a internet, permitiu indivíduos de diferentes locais interagirem por meio de conexões em rede e, consequentemente, o aumento da participação de equipes de aprendizagem colaborativa. O processo de colaboração apoiado por computador permite flexibilidade, tanto de tempo quanto de espaço, fazendo uso de ferramentas colaborativas síncronas e assíncronas (STAHL et al., 2006; CUNHA, 2009, OLIVEIRA; TEDESCO, 2010).

O suporte tecnológico presente nas ferramentas de comunicação em ambientes colaborativos permite a interação entre os indivíduos envolvidos no processo educativo, além disso, possibilitam a organização, o acompanhamento e a análise da colaboração por meio das interações (LOPES, 2007).

Estudos e pesquisas têm discutido propostas e modelos que tenham como perspectiva, formas de avaliar a interação e a colaboração nas diversas ferramentas colaborativas presentes nos ambientes CSCL. Alguns estudos, como de Gerosa (2004), Otsuka e Da Rocha (2005), Lopes (2007), Khandaker e Soh (2010), Dascalu, TrausanMatu, Dessus (2010), Lima et al. (2014), Pietruchinski e Pimentel (2015) e Ferraz, Oliveira e Hornink (2015) apontam que, por meio da criação de critérios e categorias das interações, que venham gerar dados quantitativos, é possível compor cenários em que a tecnologia pode viabilizar indicadores que, de alguma forma, mapeiem a colaboração dos usuários e entre um grupo de usuários. 
À criação de instrumentos informatizados que possibilitem mensurar e avaliar a colaboração de cada indivíduo, de modo que seja possível identificar se houve colaboração por parte dos participantes e se as informações compartilhadas durante a realização de uma atividade foram realmente significativas é importante para o processo ensino aprendizagem (ISHIKAWA et al., 2017). Uma das técnicas que podem ser aplicadas para avaliar mensagens significativas em ambientes colaborativos são as ontologias que representam e capturam um conhecimento bem fundamentado sobre o domínio. Essa fundamentação possui o intuito de proporcionar um vocabulário comum e bem estruturado desse conhecimento. Mapeamentos ontológicos podem ser utilizados para integração de dados, consulta de respostas e tarefas de análise comparativa entre ontologias (ABBAS; BERIO, 2013).

O uso de múltiplas ontologias também é abordado por Wimalasuriya e Dou (2009) para a extração de informações. A técnica utilizada pelos autores foi baseada no uso de expressões regulares para capturar informações por meio de palavras específicas, frases e características linguísticas em simples e múltiplas ontologias. Os resultados trouxeram base para afirmar as vantagens da hipótese do uso de múltiplas ontologias em extração de informações.

Este trabalho tem como objetivo apresentar uma ontologia de referência no domínio da Estatística, utilizada para fazer um mapeamento ontológico com o intuito de classificar mensagens significativas em um ambiente de aprendizagem colaborativa denominado Collabora (ISHIKAWA et al., 2018).

\section{Referencial Teórico}

Stahl et al. (2006) considera que a colaboração é conceitualizada como o processo de construção de significado compartilhado. Dessa forma, deve ser entendida como a realização da interação entre os participantes e pode ser analisada como se ocorresse durante as sequências de declarações ou mensagens dos múltiplos participantes.

Gerosa (2004) e Lopes (2007) propuseram em seus trabalhos a categorização de mensagens. A estruturação e a categorização tiveram como base o modelo de colaboração 3C e implementadas no ambiente de aprendizagem AulaNet (PUC-Rio) (GEROSA, 2004).

Lopes (2007) investigou como avaliar a aprendizagem a partir das interações dos alunos na ferramenta fórum. Desenvolveu um protótipo de um fórum categorizado semanticamente, que propôs inícios predefinidos para as mensagens e posterior análise das interações, de acordo com uma taxonomia que contempla indicadores de aprendizagem. Foram realizados dois estudos de caso e as técnicas de coleta de dados utilizadas foram: a observação, o arquivo de $\log s$ com registro das interações entre os alunos e a entrevista não estruturada. As sentenças mais representativas foram classificadas em categorias, subcategorias e fases de aprendizagem e serviram de base para o protótipo da ferramenta, que foi implementada na plataforma Pii, denominada Classificador de Mensagens (CSMG).

É consenso entre Gerosa (2004) e Lopes (2007) que a estruturação e a categorização de mensagens, além de atenuar a sobrecarga de informação de uma discussão por meio de ferramentas de comunicação, facilitam a organização e interpretação de conteúdo, diminuem os problemas de sobrecarga de informação e promovem a colaboração. No caso do modelo estruturado por Lopes (2007), o mesmo auxilia o processo de avaliação em ambientes de Educação à Distância (EAD) e permite a identificação de indicadores de aprendizagem.

Otsuka e Da Rocha (2005) exploram tecnologias computacionais de agentes e mineração de dados para apoiar a avaliação formativa de atividades de aprendizagem V. $17 \mathrm{~N}^{\circ}$ 3, dezembro, 2019 RENOTE DOI: 
colaborativas no ambiente de aprendizagem TelEduc. Foram coletados dados das interações por meio das ferramentas InterMap, que utilizam técnicas de visualização de informação para mapear a interação e a participação dos alunos e da tecnologia Acessos, que permitiu a geração de relatórios sobre os acessos dos aprendizes nos cursos.

Ferraz, Oliveira e Hornink (2015) também utilizaram os dados obtidos em logs, propondo a criação e desenvolvimento de índices de colaboração e participação do usuário na plataforma Moodle, denominada indexMoodle. Os autores selecionaram os dados relacionados com a participação (ações do usuário de acessos/visualizações) e colaboração (ações de interação) e construíram os índices globais de participação e colaboração por meio da tabela $m d l \_l o g$ em que são armazenados todos os tipos de ações possíveis no ambiente virtual de aprendizagem (AVA) Moodle. A partir dos atributos module e action, desta tabela, foi possível a realização da filtragem dos dados de acordo com o módulo, disciplina e o participante, foco da análise.

Lima et al. (2014) apresentaram o desenvolvimento do aplicativo MoodleGroups, que faz uso de um sistema multiagente, responsável por analisar os fóruns que os estudantes participam e as mensagens trocadas entre os estudantes em um AVA e apresenta as informações ao professor sob a forma de sociograma por intermédio dos dispositivos móveis.

Ainda por meio da tecnologia de agentes inteligentes, Pietruchinski e Pimentel (2015) propuseram um modelo arquitetural de sistema multiagente para ser integrado a softwares competitivos. O modelo é composto por quatro módulos específicos: Módulo Domínio (características de colaboração em jogos educacionais); Módulo Pedagógico (estratégias de desenvolvimento das características); Módulo do Estudante (armazenamento das informações pelos aprendizes/estudantes) e o Módulo de Agentes (contendo dois agentes inteligentes: individual e de grupo). O Módulo Agente é responsável por encontrar os critérios para acompanhar e avaliar as atividades de colaboração entre os estudantes. Para tanto, desenvolveram categorias e métricas que podem ser adaptadas para outros tipos de ferramentas colaborativas, principalmente aquelas em que a colaboração pode ser observada como: chat, fóruns, troca de materiais. As informações obtidas pelos agentes e armazenadas por este módulo, foram baseadas nas técnicas de avaliação de colaboração adotadas por Dascalu, Dascalu, Trausan-Matu, Dessus (2010), que apresentam o uso de um conjunto de técnicas para análise de conversas em ambientes de chat, integrando diferentes técnicas de análise de superfície de textos, de postagens em redes sociais e semântica latente (LSA); e de Khandaker e Soh (2010) que utilizaram agentes inteligentes para coletar dados das interações realizadas na ferramenta Wiki, com o intuito de formar grupos equilibrados e colaborativos.

Os trabalhos apresentados usam várias técnicas para avaliar a colaboração de alunos durante o desenvolvimento de atividades colaborativas, porém não abordam como avaliar se as mensagens pertencem ao domínio da atividade que ele está resolvendo. $\mathrm{O}$ uso de ontologias segundo Gruber (1993), pode ajudar na especificação explícita de uma conceituação referente a um domínio.

Existe uma classificação básica entre dois tipos de Ontologias de: alto nível ou de fundamentação e as de baixo nível. Segundo Degen (2001), as ontologias de fundamentação são teorias ou especificações de categorias de entidades gerais, independentes de domínio, como eventos, relações, tempo, espaço, entre outros. Enquanto que as ontologias de baixo nível, são de acordo com Guarino e Giaretta (1998), atreladas ao domínio particular do que se deseja representar. Este tipo possui entidades específicas do domínio e podem ser divididas em ontologias de: domínio, classe de aplicações e de aplicações. 
Um mapeamento ontológico pode ser visto como um provedor de soluções no cenário de pesquisas baseadas em ontologias (CHOI et al., 2006). As ontologias são vistas como um fator chave para a solução da interoperabilidade entre sistemas heterogêneos e aplicações web semântica. Com isto, o mapeamento ontológico se torna necessário para realizar a combinação de simples ou múltiplas ontologias. Mapeamentos ontológicos são comumente divididos em três categorias: mapeamento entre uma ontologia integrada global e locais, entre locais e fusão e alinhamento de ontologias. Por tanto, este trabalho propõe a criação de uma ontologia para avaliar mensagens significativas em ambientes colaborativos.

\section{Materiais e Métodos}

A construção da ontologia de referência proposta neste trabalho para o mapeamento de mensagens usou a Ontology Development 101. Esta metodologia foi criada por Noy e McGuinness, (2001) e sugere a utilização de sete passos para a criação de Ontologias na ferramenta Protégé. O primeiro passo, Determinar Escopo, é usado para determinar o domínio e o escopo de uma ontologia, identificando de forma clara o propósito e os cenários de utilização da ontologia a ser desenvolvida. Para isto, é preciso responder alguns exemplos de questões, segundo Noy e McGuineess (2001): O que abrange o domínio da ontologia?; Para que se utilizará a ontologia?; Quais questões a ontologia deverá responder?; Quem utilizará e manterá a ontologia?

O próximo passo, Considerar Reuso, é importante porque antes do desenvolvimento de uma ontologia é aconselhável verificar a existência de ontologias no domínio a ser trabalhado e que podem ser reutilizadas em um projeto. Enumerar Termos, terceiro passo, permite relacionar uma lista de termos importantes presentes no ambiente de domínio da ontologia a ser criada. A relação de termos torna-se importante para os passos subsequentes.

Através da lista de termos do passo anterior é possível definir as classes de domínio e hierarquia das mesmas. Este passo é responsável por extrair os objetos que representam uma ou mais classes. A partir de um conjunto de classes definido, é necessário organizar as classes de forma hierárquica por meio de um nível de abstração mais generalizado em direção as classes mais específicas.

O quinto passo, Definir Propriedades, utiliza a lista dos termos remanescentes e deve-se observar se eles correspondem as propriedades de dados ou de relações de classe para uma determinada classe. Definir Restrições, sexto passo, permite verificar se uma propriedade de classe é no formato de dados, tais como string ou número, por exemplo. Caso contrário, se a propriedade for do tipo relação deve-se definir a que classe a relação se refere. Considera-se restrições sobre cardinalidade e valores válidos para as propriedades. Por fim, o último passo, Criar Instâncias, permite a criação de instâncias da ontologia a partir da definição das classes, atribuindo valores as suas propriedades de dados e relações.

\section{Resultados e Discussões}

A ontologia proposta usou como base de dados os registros de um ambiente colaborativo para aprendizagem de estatística (Collabora). A arquitetura do ambiente é capaz de promover e avaliar a colaboração com o intuito de obter dados que possam ser analisados da colaboração de um grupo ou estudante (ISHIKAWA et al., 2017).

O Collabora permite que as atividades possam ser iniciadas quando dois ou mais usuários estiverem online. Ao iniciar uma atividade, uma página com o enunciado do exercício escolhido é exibida com alternativas relacionadas à resolução da atividade e os usuários podem interagir por meio de um chat para chegarem a um consenso e solucionar 
a atividade, conforme exibe a Figura 1.

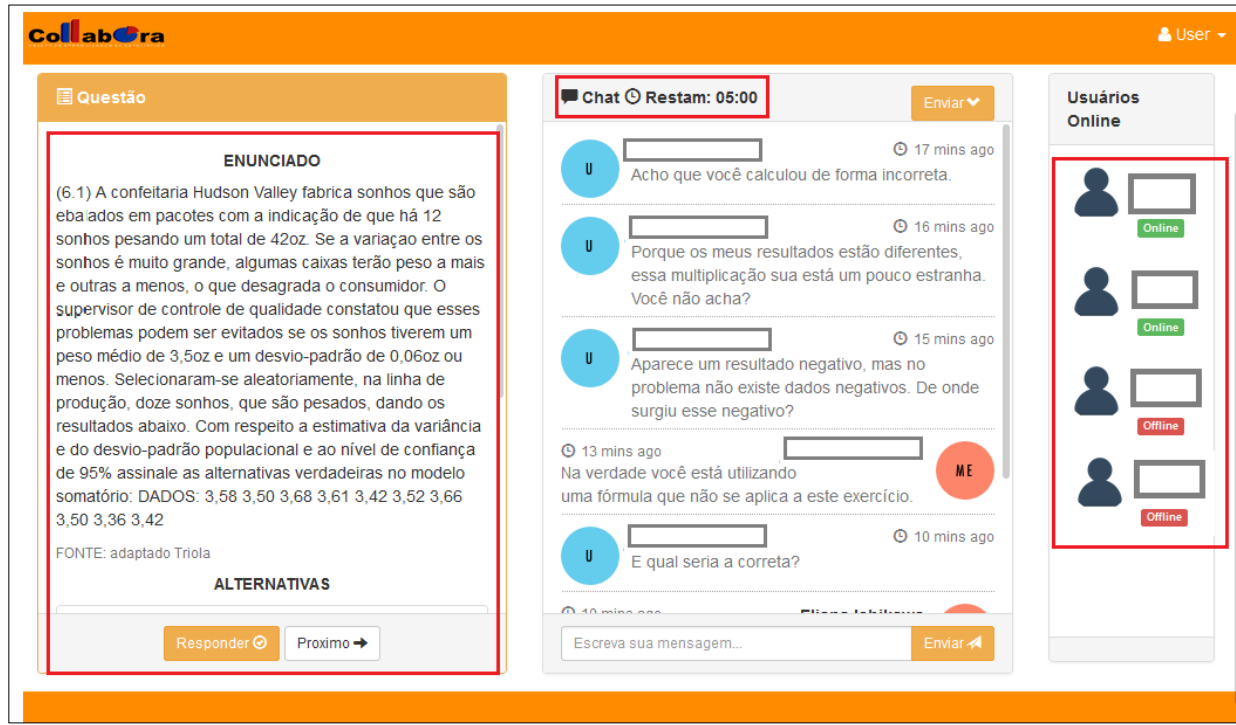

Figura 1. Exemplo de uma atividade exibe no Collabora

Os assuntos contemplados no Collabora abordam o domínio da Estatística e sua ementa contém: introdução à estatística com quatro conteúdos, a estatística para descrição, exploração e comparação de dados, estimação e testes de hipótese abordando seis conteúdos. Os assuntos totalizam 17 tipos de conteúdos que podem ser inseridos em atividades do ambiente para a resolução.

O ambiente ainda mantém um registro das interações realizadas pelos usuários e exibe na plataforma do professor para que o mesmo realize a análise qualitativa das interações realizadas pelo grupo e pelos indivíduos de forma manual, por isto a ontologia proposta neste trabalho foi desenvolvida.

O processo de criação da ontologia iniciou criando um vocabulário relacionado ao contexto da estatística para cruzamento de informações com as ontologias geradas por meio das perguntas inseridas no Collabora e interações dos participantes por meio do chat. Um exemplo do vocabulário criado é apresentado na Quadro 1.

Quadro 1- Exemplo de vocabulário criado

\begin{tabular}{|l|l|}
\hline Desvio Padrão & é a raiz quadrada positiva da variância. \\
\hline Medidas de & $\begin{array}{l}\text { é um conjunto de medidas (Amplitude, Variância e Desvio Padrão) utilizadas no } \\
\text { estudo da variabilidade de uma determinada distribuição, permitindo obter uma } \\
\text { informação mais completa acerca da "forma" da mesma. }\end{array}$ \\
\hline Variância & é a medida que permite avaliar o grau de dispersão dos valores da variável em \\
\hline
\end{tabular}

Depois foram listados os conceitos importantes relacionados ao domínio da ontologia. Dessa forma, os conceitos abstraídos para o domínio de Participação são baseados em trabalhos já existentes na literatura para a avaliação de tarefas que envolvem o diálogo entre os participantes. Neste estudo de caso o diálogo foi obtido por meio de chat no Collabora, conforme ilustra a Quadro 2.

Quadro 2 - Exemplo de mensagens trocadas entre os participantes durante a solução de uma atividade no chat do Collabora apresentada na Figura 1

\begin{tabular}{|c|l|}
\hline Aluno & Mensagem Trocadas entre os participantes \\
\hline 47 & Qual a fórmula? \\
\hline 47 & Qual o valor inicial da variância? \\
\hline 50 & O desvio padrão é de quatro \\
\hline 47 & Se a variação está entre esses valores \\
\hline 50 & Aí vc tá falando de desvio padrão ou de variância? \\
\hline 47 & Só alteraria de o valor fosse maior que 36 ou menor que 28 \\
\hline
\end{tabular}


Em um dos exemplos disponíveis para análise de mensagens do ambiente verificou-se que as mensagens significativas poderiam ser divididas entre dois tipos: mensagens que possuem relação direta com o domínio da Estatística e mensagens que representam emoções que influenciam a tomada de decisão para a resolução de um exercício. Para as mensagens com correlação com os enunciados a criação de um vocabulário no domínio da Estatística é utilizado para cruzamento de mensagens, enquanto que para mapear as mensagens que possuem sentimento, alguns marcadores linguísticos foram utilizados para realizar o cruzamento das mensagens para classificação de significância.

Para a identificação de quais marcadores linguísticos podem ser utilizados para a classificação deste tipo de mensagens utilizou-se do trabalho de David et al, (2015), que consiste na análise do diálogo entre mensagens por meio da teoria de Freire (2006). Os parâmetros utilizados para a classificação do diálogo entre participantes do Collabora foram testados em diversas plataformas e fóruns de participação. Os resultados obtidos foram essenciais para comprovar a fundamentação dos parâmetros escolhidos para a classificação.

Os pilares do diálogo foram divididos em parâmetros que se dão por marcadores linguísticos. Para este trabalho utiliza-se os parâmetros refinados e que se encaixam na proposta de aplicação da ontologia, tais como: a) afetividade; b) valorização de autonomia; c) reflexividade crítica e sua relação com o enunciado pelo conceito; e d) correlação com enunciado. Os marcadores foram definidos usando o trabalho de David et al. (2015) e são:

- Afetividade: elogios ("muito bem", "parabéns") concordância ("tem razão", "está correto"), gratidão ("obrigada", "valeu”), saudações ("olá", "bom dia”), despedida ("até mais", "tchau"), concessão ("desculpa", "perdão"), entre outros.

- Valorização de Autonomia: estímulo ("isso aí", "vá em frente"), sugestões (“eu acho que", "sugiro que”), explicação (“por que?”, "me explica?"), entre outros.

- Reflexão Crítica: opiniões pessoas (“eu acho que", "penso que”), afirmações (verbos no presente), exemplificação ("como por exemplo"), discordância ("não acha que"), entre outros.

- Correlação com Atividade: termos chave relacionados ao enunciado da atividade ou no domínio da estatística. Como por exemplo: média, mediana, valores, variância, teorema, cálculo e etc.

Com isto, criaram-se as classes da ontologia de referência: Marcadores, Mensagem e Pesos, além de uma classe denominada Collabora. A classe Mensagem é responsável por conter os parâmetros especificados anteriormente como subclasses, tais como: afetividade, correlação com o enunciado, reflexividade crítica e valorização da autonomia. A classe de marcadores representa os marcadores linguísticos e de correlação que são utilizados para classificar as mensagens em diferentes parâmetros. A classe de pesos representa o grau de cada marcador, podendo ser do tipo alto, ausente, baixo, inexistente ou médio. 
Apenas a criação de classes não fornece informações suficientes para responder as questões de competências que foram definidas. Dessa forma, foram definidos 16 marcadores linguísticos para se relacionar com a classe mensagem nas propriedades dos dados na ferramenta de ontologia Protégé. Estes marcadores são os mesmos abordados anteriormente no qual as classes foram definidas. Suas propriedades são possuir sentimentos como: afirmação, concessão, concordância, despedida, discordância, despedida, elogio, estimulo, explicação, exemplificação, gratidão, irônia, negação, opinião, saudação, sugestão ou termos matemáticos e números. Além das propriedades setadas, os objetos se relacionam com os mesmos marcadores em forma de subclasses, conforme ilustra a Figura 2.

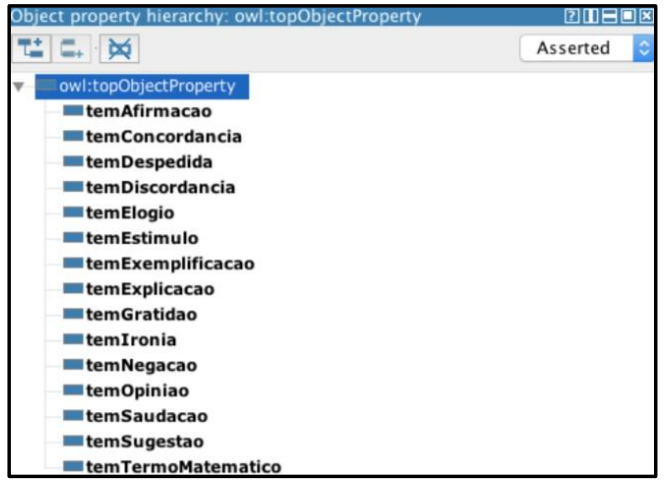

Figura 2. Configuração de propriedades da ontologia proposta

A Figura 2 apresenta as propriedades dos objetos que serão criados e caso possuam afirmação, concordância, termo matemática e entre outros. O próximo passo foi a de criação de axiomas, ou seja, as regras para a classificação das mensagens nas diferentes classes e propriedades da ontologia. Sabe-se que uma mensagem pode conter diferentes parâmetros. Desse modo, alguns axiomas foram gerados além das propriedades de relacionamentos já implementadas. Um exemplo de um axioma classificada como Significativa a uma mensagem que possui um nível de Concordância e Estímulo se encontra na Figura 3.

\section{Mensagem and (temConcordancia some owl:Thing) and (temEstimulo some owl:Thing) SubClassof Significativa}

Figura 3. Exemplo de axioma da ontologia

Um total de 42 axiomas (regras), foram gerados para fazer restrições às regras inerentes as instâncias que foram criadas e testadas na ontologia. Do total de 42 axiomas, 16 foram criados com a função de apenas classificar o tipo de mensagem a classe que ela corresponde, como exemplificado na Figura 4.

\section{Mensagem and (temNegacao some owl:Thing) SubClassOf Negacao}

Figura 4. Exemplo de um axioma padrão da ontologia

Além destes axiomas, outros 16 foram criados para classificar mensagens que já foram classificadas em alguma das classes da ontologia de referência, também classificadas como Significativas. A Figura 5 ilustra este tipo de axioma.

\section{Mensagem and (temTermoMatematico some owl:Thing) SubClassof Significativa}

Figure 5. Exemplo de um axioma para mensagem significativa da ontologia

V. $17 \mathrm{~N}^{\mathrm{o}}$ 3, dezembro, 2019 RENOTE DOI: 
Por fim, 10 axiomas, como o ilustrado na Figura 5, foram criados com operações de and (E) ou or (OU) para auxílio da classificação das instâncias. Um conjunto de instância é responsável por constituir a base de conhecimento da ontologia proposta. Um fluxo de criação de base de dados é proposto com base no funcionamento de ambientes de aprendizagem colaborativa como o Collabora. Neste caso, após a colaboração realizada dentro de um ambiente, é necessário a extração dos dados obtidos por meio do chat.

Com estes dados obtidos inicia-se o processo de criação de um dataset para realizar a aplicação da ontologia proposta a fim de analisar a correlação das mensagens com os marcadores inseridos e encontrar mensagens para construir uma base de conhecimento que pode ser utilizada e reutilizada no processo de análise de mensagens significativas. Com a extração das mensagens e análise de correlação das mesmas, por meio da ontologia de referência criada, foi possível verificar mensagens combinadas e construir uma base de conhecimento sólida para verificar a colaboração dos participantes por meio da significância das mensagens.

No intuito de testar a ontologia proposta, casos de teste foram extraídos do Collabora. O primeiro caso de teste possuia um total de 98 interações de usuários para a resolução de um exercício. Dentre essas interações, a ontologia proposta retornou um total de 33 mensagens com correlação ao domínio da Estatística e que podem ser classificadas como mensagens significativas de colaboração. A Figura 6 exibe um exemplo de mensagem sendo classificada por meio da ferramenta Protegé. A mensagem enviada pelo usuário era: "Meu desvio padrão deu 2.7".

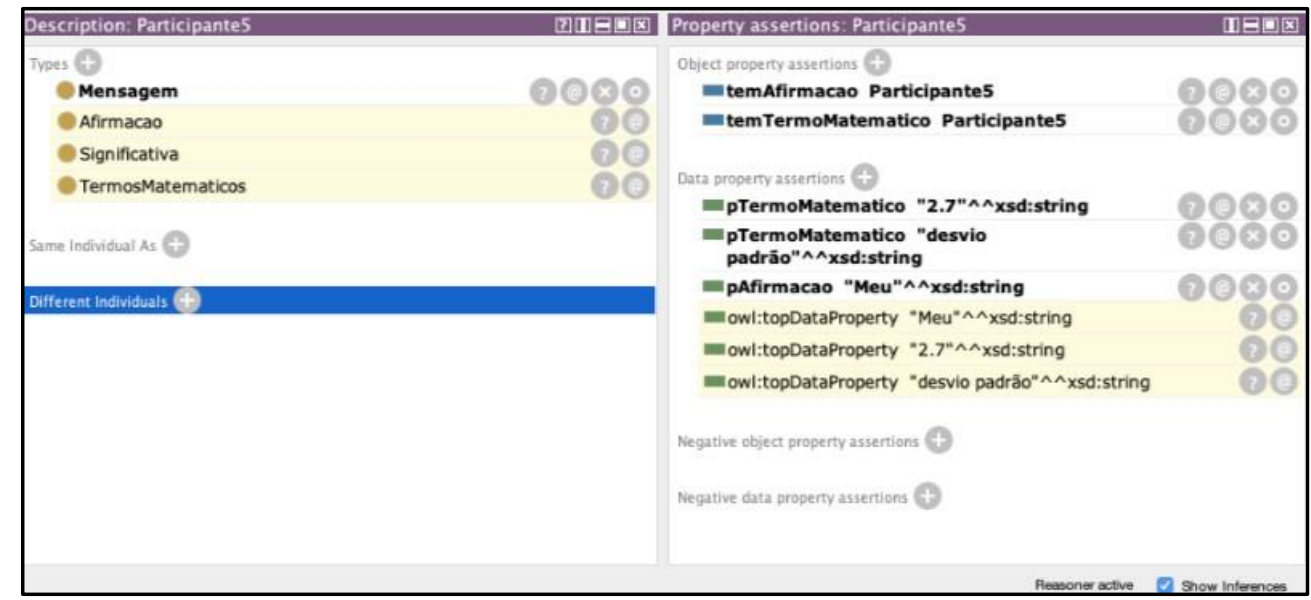

Figura 6. Exemplo de classificação de mensagem significativa

Neste exemplo, é possível verificar que a ontologia é capaz de classificar a mensagem com diferentes tipos de marcadores e associar uma relação com um exercício. A mensagem foi classificada com afirmação, termos matemáticos e assim classificada como significativa.

Em um outro caso de teste com 90 interações a ontologia proposta retornou um total de 23 mensagens significativas. Com base em alguns testes uma breve análise foi realizada e alguns indicadores são gerados para avaliação da colaboração. Estes indicadores são: a) Número de mensagens significativas alinhadas; b) Número de mensagens trocadas durante a realização de uma atividade; c) Número de parâmetros da ontologia de referência. Tais informações são úteis para diversas finalidades como: estimular a participação de alunos em um grupo de trabalho, criar escores para composição de avaliações, analisar a aprendizagem, ou outras estratégias que venham a conduzir novas e específicas abordagens para o processo educativo.

Controlar o aprendizado em um ambiente colaborativo analisando as mensagens V. $17 \mathrm{~N}^{\circ}$ 3, dezembro, 2019 RENOTE DOI: 
significativas permite verificar também a motivação dos alunos e como eles ajudam seus companheiros na solução de uma atividade. Percebe-se a melhoria das competências no pensamento crítico, uma maior capacidade em aceitar as perspectivas dos outros. $\mathrm{O}$ uso da ontologia facilita o trabalho do professor que pode obter de forma automatizada o conjunto de mensagens significativas que foram utilizadas na interação de atividades e assim determinar o nível de sua participação de cada estudante na resolução de um problema.

\section{Considerações Finais}

O uso dos recursos computacionais como uma ferramenta educacional traz vantagens quando esse é feito por grupos de alunos, uma vez que possibilita a troca de ideias entre si e entre os grupos, propiciando a construção do aprendizado coletivo e em equipe, sendo que as habilidades desenvolvidas ocorrem naturalmente, sempre sob o gerenciamento do professor (TAJRA, 2001).

À criação de instrumentos que ajudem o professor a avaliar a colaboração de cada participante durante sua troca de ideias em uma atividade colaborativa é importante para planejar o processo de ensino aprendizagem. Por isto, este artigo apresentou um mapeamento ontológico aplicado em um ambiente de atividades denominado Collabora que é capaz de avaliar a colaboração dos alunos durante a execução das atividades colaborativas.

O mapeamento foi capaz de contribuir para a avaliação das mensagens dos participantes de forma automatizada evitando a verificação e classificação manual das mensagens trocadas. A ontologia de referência proposta para o mapeamento de mensagens retorna as mensagens significativas contemplando parâmetros linguísticos e de domínio da Estatística para análise e classificação. Em relação aos testes realizados no Collabora, verificou-se que o mapeamento ontológico proposto permitiu a identificação correta das mensagens significativas trocadas entre os alunos. Trabalho que podem ser realizado a partir desta pesquisa é o refinamento do mapeamento ontológico para que contemple outras áreas do conhecimento.

\section{Referências}

ABBAS, M.; BERIO, G. Creating ontologies using ontology mappings: Compatible and incompatible ontology mappings. In: IEE/WIC/ACM International conference on web intelligente (WI) and intelligent agent technology. Proceedings... Atlanta, 2013, p. 143-146.

CHOI, N. A Survey on Ontology Mapping. ACM SIGMOD Record, v. 35, n.3, p.34- 41, 2006.

CUNHA, A. A. S. da. Definição de um modelo de sistema de aprendizagem colaborativa apoiado por computador com ênfase em trabalho em equipe. 2009. 146f. Dissertação (Mestrado em Engenharia Elétrica e de Computação) - Universidade Estadual de Campinas, Campinas (SP).

DAVID, P et al. Uma Ontologia de Domínio para a Análise do Diálogo Freireano em Fóruns de Discussão da Educação a Distância. In: XXVI Simpósio Brasileiro de Informática e Educação, Maceio, Alagoas. Anais...2015.

DASCALU, M., TRAUSAN-MATU, S., and DESSUS, P. Utterances assessment in chat conversations. Research in Computing Science, v. 46, 2010, p. 323-334.

DEGEN, W, et al. Gol: Toward an axiomatized upper- level ontology. In: ACM New York International Conference on Formal Ontology in Information Systems. Ogunquit, Maine, V. $17 \mathrm{~N}^{\mathrm{o}}$ 3, dezembro, 2019 DOI: 
Proceedings... USA, 2001, p.34-46.

FERRAZ, P O.; DE OLIVEIRA, P.; HORNINK, G.G. Desenvolvimento e Implementação de Indicadores de Colaboração e Participação no Moodle. Informática na educação: teoria \& prática, v. 18, n. 1, P. $85-95,2015$.

FREIRE, P. (2006). Pedagogia do Oprimido. Rio de Janeiro: Paz e Terra. 1a Edição: 1970.

GEROSA, M. A., FUKS, H.; LUCENA, C. J. P. Estruturação e categorização de mensagens em ferramentas de comunicação textuais assíncronas. In: WORLD CONGRESS ON ENGINEERING AND TECHNOLOGY EDUCATION. Proceedings... WCETE'2004, 2004.

GRUBER, T. Toward Principles for the Design of Ontologies Used for Knowledge Sharing. In: International Journal-Human-Computer Studies. Padova, Italy, Proceedings... Itália, 1993, p. 907-928.

GUARINO, N; GIARETTA, P. Ontologies and Knowledge Bases: Towards a terminological clarification. Towards Very Large Knowledge Bases. v.1, n.1, p.25- 32, 1998.

ISHIKAWA, E. Aplicação do Collabora como Suporte à Avaliação da Colaboração: Um Estudo na Disciplina de Probabilidade e Estatística no Ensino Superior. In: XXIX Simpósio Brasileiro de Informática e Educação. Anais... SBIE 2018.

ISHIKAWA, E; BELUZZO, L; MATOS, S; SANTOS, G. Collabora: A Colaborative Architecture for Evaluating Individuals Participation During the Development of Activities. International journal of software engineering \& applications (IJSEA), v. 8, n.1, p. 33-48, 2017.

KHANDAKER, N. and SOH, L.-K. Classroomwiki: A collaborative wiki for instructional use with multiagent group formation. Learning Technologies, IEEE Transactions on, 3(3):190$202,2010$.

LIMA, D.; et al. MoodleGroups: um Aplicativo para Identificar as Relações Sociais entre Alunos em Ambientes Virtuais de Aprendizagem. In: Symposium on Collaborative Systems. October. 2014. p. 9.

LOPES, M. S. S. Avaliação da aprendizagem em atividades colaborativas em EaD viabilizada por um fórum categorizado. 2007. 168f. Dissertação (Mestrado em Informática) - Universidade Federal do Rio de Janeiro, Instituto de Matemática, Núcleo de Computação Eletrônica, 2007.

NOY, F; MCGUINESS, L. Ontology Development 101: A guide to creating your first ontology.Disponível:

ttp://www.ksl.stanford.edu/people/dlm/papers/ontology101/ontology101-noy-

mcguinness.html, fev/2018.

OLIVEIRA, E. A. Um modelo de colaboração inteligente personalizada para ambientes de EAD. 2008. 111 f. Dissertação (Mestrado em Ciência da Computação) - Centro de Informática, Universidade Federal de Pernambuco, Recife (PE).

OLIVEIRA, E. A.; TEDESCO, P. i-collaboration: Um modelo de colaboração inteligente personalizada para ambientes de EAD. Revista Brasileira de Informática na Educação, v. 18, n. 1, p. 17-31, 2010.

OTSUKA, Joice Lee; DA ROCHA, Heloísa Vieira. Um modelo de suporte à avaliação formativa para ambientes de educação à distância: dos conceitos à solução tecnológica. RENOTE, v. 3, n. 2, 2005. 